\title{
Presencia del Cormorán Neotropical Phalacrocorax brasilianus (Gmelin, 1789) en el Valle del Motagua, Guatemala
}

\author{
Bárbara I. Escobar-Anleuํㅜ, Rita de León², José M. Mora ${ }^{3}$
}

Resumen. El cormorán neotropical (Phalacrocorax brasilianus) es un ave acuática de amplia distribución que se encuentra usualmente en aguas claras poco profundas. La especie ocupa una amplia variedad de hábitats y ha sido reportada en varias localidades de Guatemala, en zonas costeras y del interior del país. El 15 de diciembre de 2016 observamos dos cormoranes en el río Motagua, Río Hondo, Zacapa, en el valle del Motagua, Guatemala. Esta observación, así como un reporte reciente de esta especie en Baja Verapaz, sugiere que la especie está colonizando localidades que, originalmente, tuvieron diferentes tipos de hábitat y que ahora constituyen una especie de monohábitat. Esta ampliación de la distribución local del cormorán puede tener diferentes implicaciones o al menos ofrecer algunas interpretaciones en términos de la conservación de la biodiversidad.

Palabras clave. Alteración del hábitat, aves acuáticas, biodiversidad, cambio climático, conservación.

\section{Presence of the Neotropical cormorant Phalacrocorax brasilianus (Gmelin, 1789) in the Motagua Valley, Guatemala}

\begin{abstract}
The Neotropical cormorant (Phalacrocorax brasilianus) is a widely distributed aquatic bird that is usually found in shallow, clear waters. The species occupies a wide variety of habitats and has been reported from several localities in Guatemala, both in the coastal areas and the interior of the country. On 15 December 2016, we observed two cormorants at the Motagua River, Río Hondo, Zacapa, in the Motagua Valley, Guatemala. This observation as well as a recent report of this species in Baja Verapaz suggests that the species is colonizing localities that, originally, had different habitat types, but now they constitute only one type of monohabitat. This extension of the local distribution of the cormorant may have different implications or at least offer some interpretations in terms of biodiversity conservation.
\end{abstract}

Key words. Aquatic birds, biodiversity, climate change, conservation, habitat alteration.

\section{Introducción}

Phalacrocorax brasilianus (Phalacrocoracidae) es un ave suliforme de plumaje oscuro, cuello largo y cola larga (Stiles y Skutch 1995). La especie no presenta dimorfismo sexual y se distingue por exhibir un borde blanco localizado en el margen áptero posterior en la garganta. Los individuos inmaduros tienen el plumaje café oscuro, más claro en el pecho. $P$. brasilianus forma agrupaciones compuestas de hasta decenas de individuos, incluso con individuos de otras especies (Fagan y Komar 2016). El cormorán neotropical es residente en tierras bajas desde el sur de Estados Unidos, en el norte de Oklahoma y Nuevo México, hasta Argentina y Chile (Telfair y Morrison 2005). La especie se encuentra ampliamente distribuida en la región neotropical y es observada comúnmente en lagos y ríos, estuarios, granjas acuicultoras y reservorios de agua (Fagan y Komar 2016).

La ecorregión del Matorral Espinoso del valle de Motagua es considerada sobresaliente a nivel biorregional, pero está bajo un estado crítico de conservación, por lo que constituye una máxima prioridad regional (Nájera Acevedo 2006). Dicha ecorregión tiene una extensión de $2,363 \mathrm{~km}^{2}$ y contiene las zonas de vida Monte Espinoso Subtropical y el Bosque Seco Subtropical (Dinerstein et al. 1995). El río Motagua corre en la falla del Motagua que atraviesa Guatemala en el segmento sur-oeste.

La zona es considerada rica en diversidad animal y vegetal, así como en endemismos, como resultado de la combinación de condiciones climáticas influenciadas

\footnotetext{
1 Escuela de Biología, Universidad de San Carlos de Guatemala, Edificio T-10, Ciudad Universitaria, Zona 12, Ciudad de Guatemala, Guatemala. Correo electrónico bisa e8a9@hotmail.com

2 Universidad del Valle de Guatemala, Ciudad de Guatemala, Guatemala. Correo electrónico rita.dl.osorio@gmail.com

${ }^{3}$ Carrera de Gestión Ecoturística, Sede Central, Universidad Técnica Nacional, Alajuela, Costa Rica. Correo electrónico josemora07@gmail.com
} 
por los océanos Atlántico y Pacífico (Nájera Acevedo 2006). La actividad sísmica de la región, junto con las características mencionadas, ha resultado en la disponibilidad de hábitats que la caracterizan con respecto a su papel en la limitación de distribuciones y la separación de subespecies aviares que habitan al norte y sur de sus márgenes (Fagan y Komar 2016).

\section{Descripción y Discusión}

El 15 de diciembre de 2016 observamos dos individuos de $P$. brasilianus en el río Motagua, en Río Hondo (Figura 1), Zacapa, Guatemala $\left(15^{\circ} 0^{\prime} 21.51^{\prime \prime} \mathrm{N}\right.$, $\left.89^{\circ} 37^{\prime} 35.16 \mathrm{\prime W}\right)$. Aunque la especie es categorizada de común a abundante, su distribución en el país se limita al norte (Petén y Alta Verapaz), noroccidente (parte de Quiché), la costa atlántica y pacífica, así como en localidades de Huehuetenango y Sololá (Fagan y Komar 2016). Esto corresponde con lo reportado en Global Biodiversity Information Facility (GBIF), donde las localidades más cercanas a esta observación son Izabal y Alta Verapaz, a 48 y $45 \mathrm{~km}$, respectivamente (Figura 1).

Esta es la primera observación del cormorán neotropical en el valle del Motagua. Los dos individuos observados estaban posados sobre rocas en el río (Figura 2). Este sustrato rocoso tenía alguna vegetación e incluso basura y solo sobresalía unos 40 $\mathrm{cm}$ sobre el nivel del agua. Se pudo observar a los cormoranes por unos cinco minutos antes de que se alejaran volando; no se constató permanencia de los individuos en el área.

Según la clasificación de zonas de vida de Holdridge, el valle del Motagua es uno de los pocos sitios de Bosque muy Seco Tropical de Centroamérica. A excepción de los bosques de galería, en los que el flujo constante de agua permite el desarrollo de comunidades que se mantienen verdes aún en la época seca, las partes bajas están dominadas por especies espinosas como cactáceas, Vachellia y otras leguminosas (Powell y Palminteri 2002).

Estas condiciones han aislado a algunas poblaciones que han resultado en endemismos en animales y plantas. No obstante, el hábitat en general ha sido alterado o destruido en varios lugares para dar paso al desarrollo socioeconómico (Nájera Acevedo 2006). La contaminación en la ribera es evidente, con presencia de desechos de materiales de diversos

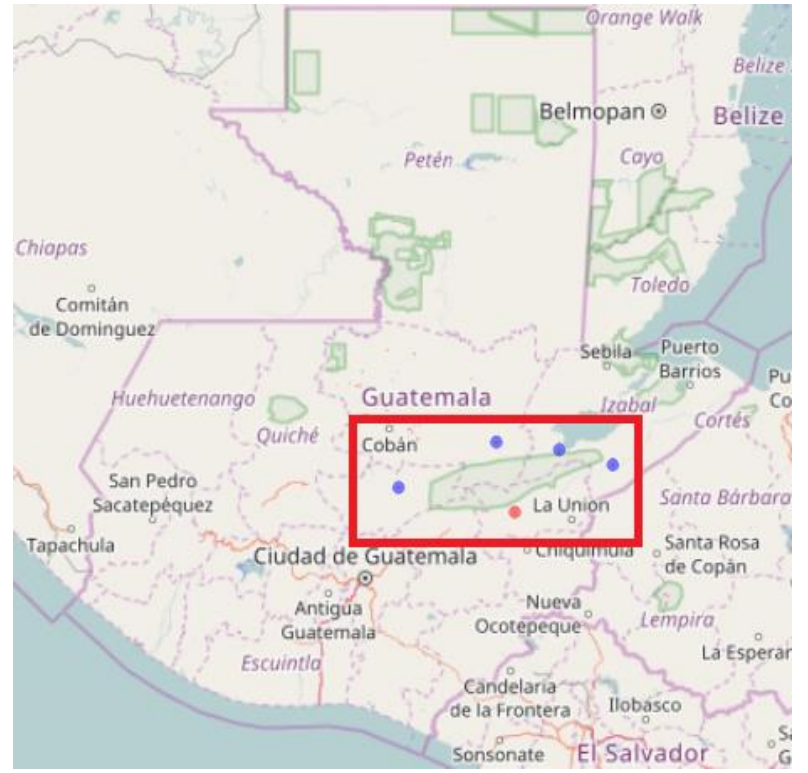

orígenes y desechos arrastrados por la corriente del río (Figura 3).

Figura 1. Localidad de observación de Phalacrocorax brasilianus en el valle del Motagua, Zacapa, Guatemala (punto color rojo). Los puntos color azul son las localidades más cercanas reportadas en eBird y GBIF. Mapa elaborado por Bárbara Escobar.

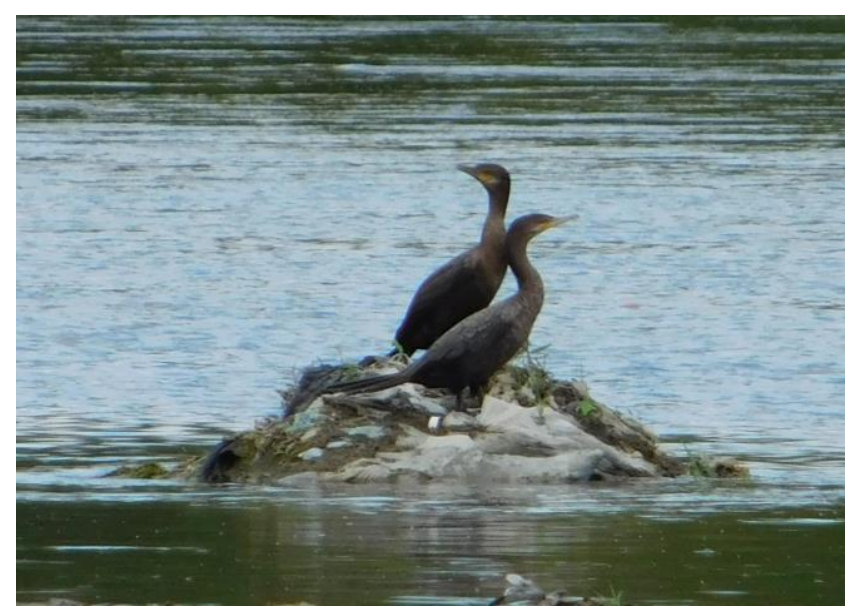

Figura 2. Cormoranes (Phalacrocorax brasilianus) en el río Motagua, Río Hondo, Zacapa, Guatemala. Foto: Bárbara Escobar (Diciembre 15 de 2016). 


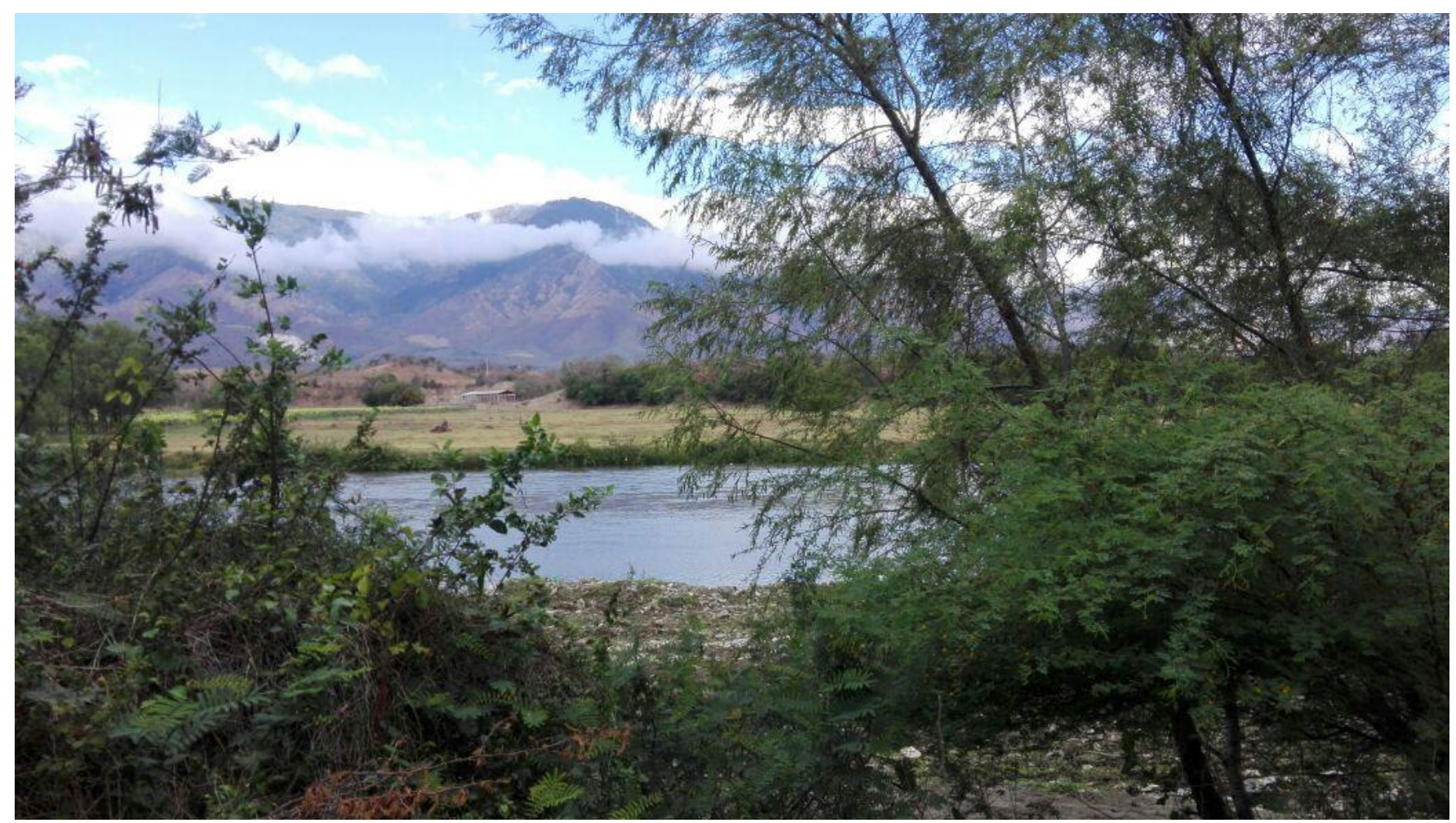

Figura 3. El río Motagua en el valle de Zacapa, Guatemala, a la altura de Río Hondo corre entre sitios perturbados que incluye alguna vegetación remanente (primer plano). Nótese la acumulación de basura en la ribera del río. Fotografía por Bárbara Escobar (15 de diciembre de 2016).

Alberto Larios subió al portal de eBird un avistamiento de Phalacrocorax brasilianus en Baja Verapaz el 23 de enero de 2017. Aunque la distancia entre este punto y el aquí reportado (unos $80 \mathrm{~km}$ ) podría ser recorrida por los mismos individuos, es importante resaltar las distintas condiciones del hábitat de reportes previos, como el que aquí se presenta para el valle del Motagua, lo cual tiene implicaciones importantes en términos de conservación general de la biodiversidad. Diversos aspectos, incluido el cambio climático, habrían transformado la diversidad de ecosistemas en un monohábitat (la homogeneización de lo que antes fue un paisaje heterogéneo).

Estos registros no representan en sí una extensión del ámbito de distribución general del cormorán neotropical, pero tienen la particularidad de ser localidades nuevas para la especie lo que extiende su ámbito de ocupación local. Ambas observaciones denotan el carácter generalista de la especie en términos de hábitat y posiblemente alimentación. Este hecho indica el nivel de homogeneización del hábitat que ha resultado de las actividades humanas y la presencia de la especie en estas localidades es relevante debido principalmente a una probable indicación de una situación de desequilibrio ecológico. Algunas especies de aves podrían tener condiciones de preadaptación para colonizar este tipo de ecosistemas o áreas resultantes de ese proceso de homogneización.

Varias especies de aves han colonizado nuevas localidades y las razones de estas invasiones pueden tener relación con las alteraciones del hábitat y el cambio climático (Mora y López 2014). Aparentemente, el cormorán ha ido ocupando paulatinamente nuevas localidades (Stiles y Skutch 1995). La pregunta inmediata es ¿qué ha mediado para que el cormorán esté aumentado su distribución en ocupación de nuevas localidades? Se conocen los efectos negativos sobre la biodiversidad que tienen algunos factores como la destrucción del hábitat, la fragmentación y la sobreexplotación. Sin embargo, en varios casos, la explicación de las ampliaciones de la distribución de algunas especies no es tan clara (Mora y López 2014). 
El cambio de la distribución de muchas especies de animales y plantas provoca una redistribución de la vida en la Tierra lo cual tiene un gran significado ecológico (Sunday et al. 2012). Las tasas de sustitución de especies en las comunidades ecológicas pueden resultar en disturbios severos (Mora y López 2014). Una reformulación de las comunidades de especies podría resultar en numerosas extirpaciones locales y extinciones (Root et al. 2003). En el valle del Motagua es difícil cualquier predicción en este sentido debido a la poca información acerca de las comunidades de aves del lugar. Referente a las aves acuáticas, solo se conoce la presencia de cuatro especies, más tres especies ribereñas tipo correlimos (Valle et al. 1999). Estos autores anotaron un total de 104 especies de aves para el valle. Es imprescindible actualizar esta lista y seguir los patrones de movimiento de las aves acuáticas para entender mejor la dinámica de la comunidad de aves en el valle de Zacapa. Nuestro reporte de la presencia del cormorán neotropical en el valle del Motagua como nuevo sitio de avistamiento es importante porque puede ser un indicador de las modificaciones y el impacto que las actividades antropogénicas han causado en los ecosistemas. Para tener más claridad sobre la situación general es necesario generar información sobre aspectos del valle del Motagua, incluidas la disponibilidad de alimento para aves piscívoras y la competencia interespecífica por este tipo de recurso.

Agradecimientos. A Oscar Sacahuí Reyes por sus aportes, su apoyo, buena actitud y apertura para la publicación de esta nota. A Daniel Germer por sus observaciones y sugerencias.

\section{Literatura Citada}

eBird. 2016. An online database of bird distribution and abundance [web application]. eBird, Cornell Lab of Ornithology, Ithaca, New York. Disponible: http://www.ebird.org. (Accedido el 20 de marzo de 2017).

Dinerstein, E., D.M. Olson, D.J. Graham, A.L. Webster, S.A. Primm, M.P. Bookbinder y G. Ledec. 1995. Una evaluación del estado de conservación de las ecorregiones terrestres de América Latina y el Caribe. Banco Mundial. Fondo Mundial para la Naturaleza. 134 p.
Fagan, J. y O. Komar. 2016. Field Guide to Birds of Northern Central America. Peterson Field Guides, New York: Houghton Mifflin Harcourt. 438 p.

GBIF.org. 2016. GBIF Occurrence. Disponible: http://doi.org/10.15468/dl.9nqr8I (Accedido 23 de diciembre de 2016).

Mora, J.M. y L.I. López. 2014. Cambios en la Distribución de las Aves: ¿Qué puerta Hemos Abierto?; los Casos del Chico Piojo (Campylorhynchus rufinucha) y la Paloma Ala Blanca (Zenaida asiatica) en Costa Rica. Ceiba 52(2):230-236.

Nájera Acevedo, A. 2006. The conservation of the thorn scrub and dry forest of the Motagua Valley, Guatemala: promoting the protection of a unique ecoregion. Lyonia $9(2): 7-19$.

Powell, G. y S. Palminteri. 2002. Terrestrial ecoregions: deserts and xeric shrublands. Wild World Fund. Disponible:

http://www.worldwildlife.org/ecoregions/nt1312 (Accedido 28 de diciembre de 2016).

Root, T.L., J.T. Price, K.R. Hall, S.H. Schneider, C. Rosenzweigk y J.A. Pounds. 2003. Fingerprints of global warming on wild animals and plants. Nature 421(6918):57-60.

Sunday, J. M. A.E. Bates y N.K. Dulvy. 2012. Thermal tolerance and the global redistribution of animals. Nature Climate Change 2(9):686-690.

Telfair II, R.C. y M.L. Morrison. 2005. Neotropic Cormorant (Phalacrocorax brasilianus), The Birds of North America. En P.G. Rodewald (Ed.). Cornell Lab of Ornithology, Ithaca. Disponible: https://birdsna.org/SpeciesAccount/bna/species/neocor (Accedido el 15 de mayo de 2017).

Valle L., R. Soto, M. del P. Negreros y C. Castañeda. 1999. Áreas prioritarias para la conservación en el sector norte del matorral espinoso del Valle del río Motagua, Guatemala. Fundación Defensores de la Naturaleza, Programa Ambiental Regional para Centroamérica/Central American Protected Area System, Guatemala. $168 \mathrm{p}$.

Recibido para publicación el 11 de agosto del 2017.

Aceptado para publicación el 1 de octubre del 2017.

Publicado el 12 de febrero del 2018. 